\title{
Design of High-Area-Ratio Nozzle Contours Using Circular Arcs
}

\author{
K. Schomberg* and J. Olsen‡ \\ UNSW Australia, Sydney, New South Wales 2052, Australia \\ and \\ G. Doig辛 \\ California Polytechnic State University, San Luis Obispo, California 93407
}

DOI: $\underline{10.2514 / 1 . B 35640}$

\begin{abstract}
A method using circular arcs to generate the divergence contour in a supersonic nozzle is presented. Comparison of the arc-based geometry with existing nozzle contours demonstrated that an average decrease in axial length of 7.5\% can be expected when the arc-based design method is applied to a core stage nozzle. Two arc-based and conventional nozzles were evaluated numerically across the pressure operating range of a core stage engine to compare calculated thrust and separation characteristics with existing data. The length-weighted thrust coefficient was increased by $0.3-1.8 \%$ in the arc-based design in both configurations. Separated flow characteristics were compared using contours of Mach number and static pressure distributions, which suggested equivalent side loading in the arc-based nozzle at separated flow conditions. The result indicates that a geometric advantage independent of thrust may be achieved when the arc-based method is applied to high-area-ratio nozzle contour design.
\end{abstract}

\section{Nomenclature}

$b \quad=\quad$ number of arc segments within curve

$C_{F}=$ thrust coefficient

$C_{F, w}=$ weighted thrust coefficient

$C_{x}=$ nozzle length-weight coefficient

$E=$ numerical error

$k=$ turbulent kinetic energy, $\mathrm{m}^{2} \mathrm{~s}^{-2}$

$L=$ total contour axial length, $\mathrm{m}$

$M a=$ Mach number

$P \quad=\quad$ static pressure, $\mathrm{Pa}$

$r \quad=$ radius, $\mathrm{m}$

$T_{W}=$ thrust-to-weight ratio

$x=$ horizontal distance, $\mathrm{m}$

$y=$ vertical distance, $\mathrm{m}$

$\varepsilon=$ turbulent dissipation rate, $\mathrm{m}^{2} \mathrm{~s}^{-3}$

$\theta \quad=$ angle, deg

$\omega=$ specific dissipation rate, $\mathrm{s}^{-1}$

Subscripts

$\begin{array}{ll}a & =\text { arc nozzle, turning curve } \\ C & =\text { coarse mesh } \\ c & =\text { conical nozzle, turning curve } \\ e & =\text { nozzle exit } \\ F & =\text { fine mesh } \\ i & =\text { arbitrary contour point } i \\ j & =\text { arbitrary contour point } j \\ m & =\text { inflection point, arc contour } \\ n & =\text { endpoint, arc contour } \\ S & =\text { standard mesh } \\ t & =\text { nozzle throat }\end{array}$

Presented as Paper 2014-3999 at the 50th AIAA/ASME/SAE/ASEE Joint Propulsion Conference, Cleveland, OH, 28-30 July 2014; received 21 October 2014; revision received 14 June 2015; accepted for publication 15 June 2015; published online 4 September 2015. Copyright () 2015 by Kyll Schomberg. Published by the American Institute of Aeronautics and Astronautics, Inc., with permission. Copies of this paper may be made for personal or internal use, on condition that the copier pay the $\$ 10.00$ per-copy fee to the Copyright Clearance Center, Inc., 222 Rosewood Drive, Danvers, MA 01923; include the code 1533-3876/15 and $\$ 10.00$ in correspondence with the CCC.

*Ph.D. Candidate, School of Mechanical and Manufacturing Engineering; kyll.schomberg@unsw.edu.au. Student Member AIAA.

'Lecturer, School of Mechanical and Manufacturing Engineering.

¥Assistant Professor, Aerospace Engineering Department; also Adjunct Lecturer, UNSW Australia, Sydney, New South Wales 2052, Australia. Senior Member AIAA. $\begin{array}{lll}0 & = & \text { stagnation condition } \\ 1 & = & \text { inflection point, conventional contour } \\ 2 & = & \text { endpoint, conventional contour }\end{array}$

\section{Introduction}

$\mathbf{T}$ HE function of a supersonic nozzle is to convert the thermal energy of the combustion products into kinetic energy in order to produce thrust. In rocket propulsion systems, the propellant is stored on board and ejected at high velocity in a momentum exchange process. Because the propellant is carried within the system, it is desirable to maximize the thrust efficiency or specific impulse of the propulsion system to minimize vehicle mass. This process involves accelerating the combustion products to supersonic velocities through the use of a convergent-divergent (CD) nozzle.

The simplest divergent contour uses a constant angle to increase the nozzle area ratio, resulting in a conical nozzle for an axisymmetric design. The primary benefit to a constant angle design is that thrust and flow parameters can be approximated using quasi-one-dimensional flow theory. However, a correction is required due to the nonaxial nature of the exhaust velocity and gives rise to thrust losses that are a function of the nozzle wall angle [1]. An ideal divergence contour that produces uniform and axial flow at the nozzle exit via isentropic turning of the nozzle flow can be achieved through use of the method of characteristics (MOC) [2].

Although an ideal contour is capable of producing a uniform axial exit velocity, the excessive length required to turn the flow without inducing shocks renders an ideal contour unsuitable for weightsensitive applications such as orbital launch systems. In an ideal design, the contour extends throughout the nozzle in such a way that the wall angle decreases as the flow is progressively turned. Therefore, if a small divergence angle is accepted, the nozzle length can be reduced through the use of a truncated ideal contour (TIC) [3]. To further reduce nozzle length, and therefore overall system weight, an axially compressed truncated ideal contour (CTIC) can be used [4] . In a CTIC, the severity of the contour is increased in the turning section, which increases the strength of the compression waves, resulting in the formation of a shock if these waves coalesce.

Instead of manipulating an ideal contour to minimize length, a unique contour can be generated to maximize thrust for a prescribed length using the calculus of variations [5]. This was later simplified by Rao and is often denoted as a thrust-optimized contour (TOC) [6]. As a TOC can only be described through a list of contour points, Rão derived an approximation through use of a skewed parabola [7]. This contour variant is referred to as a thrust-optimized parabola (TOP) and will result in a lower thrust than an equivalent TOC. However, direct optimization studies of TOP contours have shown that the 


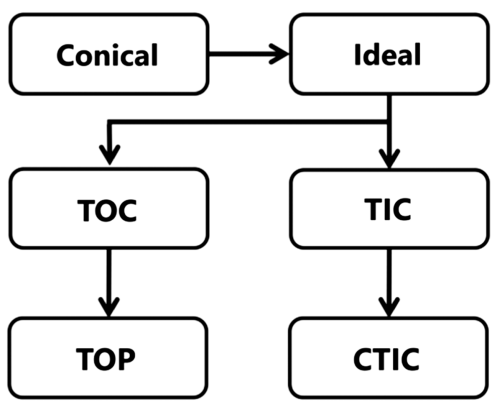

Fig. 1 Historical design progression of the supersonic nozzle divergence contour.

difference in thrust can be less than $0.2 \%$ compared to an equivalent TOC [8]. Additionally, the spatial description of the TOP contour naturally facilitates a much greater degree of flow control. This can be used to increase nozzle area ratio, and therefore vacuum performance through the manipulation of static wall pressure [9]. The historical progression of nozzle contour design is outlined in Fig. 1.

Although the TOP and compressed truncated ideal contours are a derivative of a design generated using the MOC, the resulting flowfield is neither ideal nor thrust optimized. Observation of the historical progression of nozzle design therefore suggests that the current requirements for high-area-ratio nozzles are independent of the inherent characteristics within conventional design methods. Because of this, an alternative design method was investigated to determine the feasibility of an unconventional approach toward the generation of a rocket nozzle contour.

The design method selected in this work was based on the use of a finite series of circular arcs for the construction of the nozzle contour. Focus was placed on generation of the divergence contour, as it is widely accepted that any smooth curve is sufficient for use in the convergent section of the nozzle [10]. The concept of using circular arcs to generate the divergence contour in a supersonic nozzle was proposed soon after the application of CD nozzles to rocket motors [11]. Unfortunately, any documented analysis of arc-based contours for use in rocket nozzle applications is limited within the public domain $[11,12]$. However, one study compared the thrust between a nozzle constructed from circular arcs to both a TOC and TOP design and suggested the difference between contour generation methods was low for a nozzle expansion ratio of 12-35 using air as the working fluid [13]. In the present work, the feasibility of an arc-based design method for generation of high-area-ratio nozzle contours has been determined using a numerical approach across the range of flow regimes expected to occur in a core stage rocket engine. Viability of the arc-based design method was subject to a reduction in contour length, independent of calculated thrust across the entire range of operating conditions.

\section{Design}

The major requirement for the design was that the general flow characteristics were consistent with a conventional nozzle in a geometrically favorable design. A reduction in contour length for consistent area ratio may increase the thrust-to-weight ratio of the nozzle and would imply a reduction in engine weight and cooling requirements. The production of a contour that could be described spatially was desirable, as it would enable greater ease of nozzle scaling both geometrically and for varying gas compositions compared to a TOC/ TIC design.

\section{A. Methodology}

The design method uses a series of circular arcs to achieve the contour design requirements. Similar to conventional nozzle design, the divergence contour was separated into an expansion curve and a turning curve, where each curve can be described by a finite series of circular arcs. The use of arcs to generate the divergence contour enables a full description of the curve through the manipulation of trigonometric relations. The spatial description of the turning contour is achieved by deconstructing each arc segment into two right-angled triangles. This process is shown in Fig. 2.

To ensure the inherent capability of geometric scaling, all contour parameters can be normalized by the throat radius, as shown in Eq. (1). In all contours designed using the arc-based design method, any two arbitrary points on each arc segment will be connected by a common radius. The equation for the radius connecting any two arbitrary points $i$ and $j$ is shown in Eq. (2):

$$
\bar{r}=\stackrel{r}{r_{t}}, \quad \bar{x}=\stackrel{x}{\leftarrow} r_{t}, \quad \bar{y}=\frac{y}{r_{t}}
$$

$$
\bar{r}=\frac{\overline{y_{j}}-\overline{y_{i}}}{\left|\cos \theta_{j}-\cos \theta_{i}\right|}=\frac{\overline{x_{j}}-\overline{x_{i}}}{\left|\sin \theta_{j}-\sin \theta_{i}\right|} \leftarrow
$$

It can be seen in Eq. (2) that the radius between two points is dependent on the angle between these points as well as either the horizontal or vertical distance. As the finite series of circular arcs is based on a space-marching scheme, it is assumed that the spatial conditions at point $i$ will be known. The position at $j$ must then be iteratively defined for the contour to be generated. The vertical position can be determined with respect to the required area ratio and, similarly, the horizontal position determined with respect to a prescribed nozzle length. The angle at $j$ can then be selected by the designer, as long as this value obeys the inequality defined in Eq. (3). It is worth noting that, in a conventional nozzle, the tangent of the contour at the throat will be parallel to the nozzle axis. However, the arc-based design method can naturally extend to unconventional nozzle concepts that use a nonaxial throat:

$$
\theta_{t}, \theta_{n} \leq \theta_{j}<\theta_{i} \leq \theta_{m}
$$

As long as the wall angle is known, a complete spatial description of the contour will be possible. The horizontal and vertical positions of the contour for any angle can be calculated using Eqs. (4) and (ㅁ), respectively. The wall angle can then be incrementally changed to describe the contour. Comparatively, the corresponding wall angle can be calculated for any nondimensional length value, as shown in Eq. (6) for nozzle length. This capability is of particular importance when considering flow properties in a scaled variant of the nozzle contour:

$$
\begin{aligned}
& \bar{x}=\overline{r_{i j}}\left(\sin \theta_{i}-\sin \theta\right)+\overline{x_{i}} \quad \forall \theta_{j} \leq \theta \leq \theta_{i} \\
& \bar{y}=\overline{r_{i j}}\left(\cos \theta-\cos \theta_{i}\right)+\overline{y_{i}} \quad \forall \theta_{j} \leq \theta \leq \theta_{i}
\end{aligned}
$$

$$
\theta=\sin ^{-1}\left[\left(1-\frac{x}{L}\right) \sin \theta_{i}+\frac{x}{L} \sin \theta_{j}\right]
$$

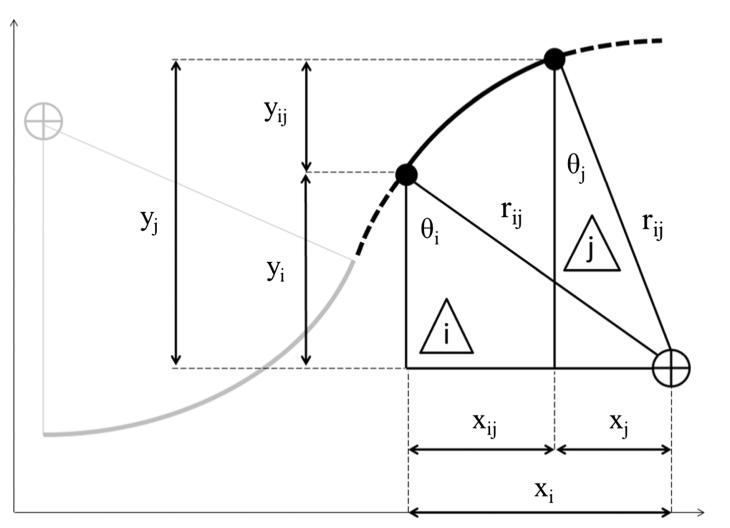

Fig. 2 Spatial description of an arc segment in the turning curve. 
The nozzle contour can be described by the sum of the horizontal and vertical or radial arc segments. The limits of these values are likely to be a design requirement of the nozzle itself; and, as such, the number of segments and angles between segments must be selected by the designer. Calculations of these values are subject to Eq. (3) and can be determined using Eqs. (7) and (ㅁ):

$$
\begin{aligned}
& \overline{x_{t n}}=\sum_{i=0}^{b} t_{i j}\left(\sin \theta_{j}-\sin \theta_{i}\right) \leftarrow \\
& \overline{y_{t n}}=\sum_{i=0}^{b} t_{i j}\left(\cos \theta_{j}-\cos \theta_{i}\right) \leftarrow
\end{aligned}
$$

\section{B. Length Reduction}

The length of any supersonic nozzle contour is often compared to the length of an equivalent constant angle contour [10]. Assuming that the expansion curve is consistent between both contours, a direct comparison can be made between the turning curves. The length of a constant angle contour is a function of the required vertical or radial distance and contour angle, as shown in Eq. (9). Comparatively, the turning curve length of a nozzle generated using the arc method can be determined through manipulation of Eqs. (ㄱ) and ( $\underline{8})$ and is given in Eq. (10). When defining a ratio between the nozzle lengths determined through the circular arc method and that of a constant angle contour, the required vertical of radial distance is cancelled. The ratio becomes a function of wall angle only, as shown in Eq. (11):

$$
\begin{gathered}
L_{c}=\frac{y_{m n}}{\tan \theta_{c}} \\
L_{a}=\left(y_{m n}\right) \frac{\left(\sin \theta_{m}-\sin \theta_{n}\right)}{\left(\cos \theta_{n}-\cos \theta_{m}\right)} \leftarrow \\
\frac{L_{a}}{L_{c}}=\tan \theta_{c} \frac{\left(\sin \theta_{m}-\sin \theta_{n}\right) \overleftarrow{\approx}}{\left(\cos \theta_{n}-\cos \theta_{m}\right)} \frac{2 \theta_{c}}{\theta_{m}+\theta_{n}}
\end{gathered}
$$

If a constant angle is selected by the designer to produce a baseline nozzle length, the length ratio then becomes a function of the expansion angle and the exit or divergence angle of the circular arc contour. This length ratio can also be approximated as a simple power function for the given constant angle, as shown in Eq. (11). The difference between the exact and approximated length ratios and a contour plots of the rate of length reduction are shown in Figs. $\underline{3}$ and 4 , respectively, for a baseline constant angle contour of $15 \mathrm{deg}$.

A difference of $5 \%$ or less between the exact and approximate length ratios was observed across the range of practical angles for an axial-throat nozzle. Variation of the exact function can be seen to increase as the difference between the combined design angle and a value of twice the selected conical angle increases, where a maximum variation of $5 \%$ was observed at the upper limit of the angles considered here. It can be seen from Fig. 4 that a decreasing exponential trend in length ratio was evident, and this trend was equally weighted between the expansion and divergence angles with respect to the magnitude of length reduction. It can be seen from the contour plot that a considerable reduction in nozzle length (i.e., less than the $80 \%$ bell [10]) is restricted to high-angle designs. This restriction may account for the lack of interest in an arc-based contour for use in early nozzle design $[11,12]$, as the low area ratios used inherently preclude the use of high angles in contour generation.

\section{Parametric Study}

To provide an initial estimation of the viability for the arc-based design method, a preliminary analysis was conducted to verify that a reduction in nozzle length relative to a conventional design would be possible. A parametric study of existing contours was used to

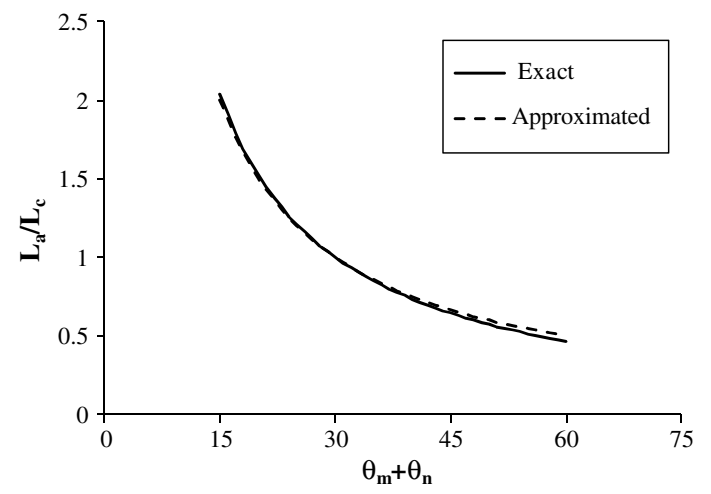

Fig. 3 Variation in exact and approximated length ratio.

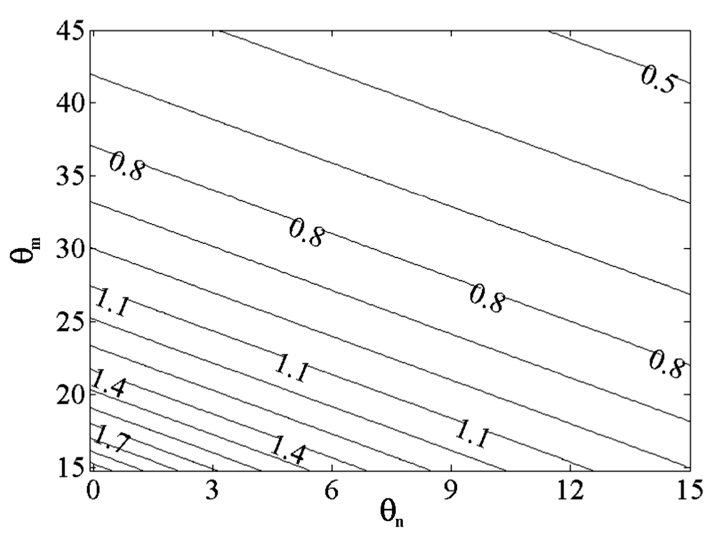

Fig. 4 Contour plot of length ratio magnitude.

compare geometric parameters of the Space Shuttle Main Engine (SSME) [9], Vulcain 2 [14], and conventional Vulcain [15] nozzles against an equivalent contour generated using the arc-based method. It is important to note that the expansion curve was kept consistent with the current design of each nozzle and a single curve was used for the turning section to give a baseline comparison of the arc design. Tables 1 and 2 outline the current specifications of conventional nozzles and $\bar{a}$ comparison with the arc-based contour length, respectively.

The arc-based contour returned a lower axial nozzle length when compared to all conventional designs, where the magnitude of this reduction increased with respect to nozzle area ratio (AR) in all cases. The associated change in thrust-to-weight ratio was calculated using the length ratio and nozzle length-weight coefficient as shown in Eq. (12):

$$
T_{W}=\overleftarrow{1} \frac{1}{1-C_{x}\left(1-x_{n} / x_{2}\right)} \leftarrow
$$

Table 1 Geometric parameters in conventional nozzle designs

\begin{tabular}{lcccccc}
\hline \hline Model & $y_{t}$ & $\overline{r_{t 1}}$ & $\overline{y_{2}}$ & $\theta_{1}$ & $\theta_{2}$ & $\overline{x_{2}}$ \\
\hline Vulcain & 0.1312 & 0.500 & 6.716 & 35.025 & 6.500 & 15.74 \\
Vulcain 2 & 0.1370 & 0.500 & 7.629 & 37.000 & 5.500 & 18.62 \\
SSME & 0.1309 & 0.392 & 8.803 & 37.000 & 5.300 & 23.52 \\
\hline \hline
\end{tabular}

Table 2 Comparison of arc-based and conventional nozzle contour length

\begin{tabular}{lccc}
\hline \hline Model & $\overline{x_{n}}$ & $\Delta L, \%$ & $\Delta T_{W}, \%$ \\
\hline Vulcain & 15.13 & -3.910 & 0.389 \\
Vulcain 2 & 17.09 & -8.220 & 0.829 \\
SSME & 20.20 & -14.12 & 1.432 \\
\hline \hline
\end{tabular}


A nozzle length-weight coefficient of 0.1 was selected for this study based on the nozzle-to-engine weight fraction of 0.13 and 0.135 in the SSME [16] and Vulcain [17] engines, respectively. A value of 0.1 would yield a conservative estimate of the expected improvement while taking into account the difference between actual contour length and axial distance, which was found to be less than $0.5 \%$ for the design cases considered here. Although a performance increase on the order of $0.3-1.5 \%$ may seem trivial, the increase in thrust coefficient as a result of the Vulcain 2 upgrade was on the order of $1 \%$ [14].

\section{Design Case}

To explore the performance characteristics of the arc-based design method, two scaled variants of the axisymmetric Vulcain nozzle were selected for analysis. The nozzle variants were based on a scaling procedure geared toward adaptation of the contour using a nondimensional length parameter. In these cases, the length parameter selected was the radius at the nozzle exit (S1) and throat (S3). Both the S1 and S3 nozzles have previously been validated and shown to adequately represent flow structure within the Vulcain nozzle [15]. Table $\underline{3}$ outlines the geometric parameters for the $\mathrm{S} 1$ and $\mathrm{S} 3$ nozzles and the corresponding reduction in contour length as a result of the arc-based design method.

The expansion contour for both nozzles was kept consistent with that used in [15]. The arc-based approach to the turning curve again resulted in a reduction in overall nozzle length when compared to the existing design. Although the area ratio between the S1 and S3 nozzles was similar, the magnitude of the length reduction in the S3 nozzle was much greater. For simplicity, the turning curve was kept to a single arc, allowing the wall angle to be described with respect to the nondimensional length of the nozzle contour, as shown in Eq. (6). With the wall angle known, both arc-based contours could be described spatially throughout the domain. It is important to note that the design of all nozzle turning contours in the analysis was restricted to a single circular arc, where a series of arcs may produce a greater magnitude of length reduction. Based on the parametric study and design case, it could be concluded that the arc-based design method can produce a geometrically favorable contour for a high-area-ratio nozzle.

\section{Numerical Model}

A numerical approach was employed to assess flow behavior and calculate thrust across the flow regimes expected to occur in a core stage engine. All results were generated using the commercially available ANSYS Fluent 14.5 finite volume code. An axisymmetric pressure-based momentum-coupled solver was selected to generate all results. Although initially a subsonic flow solver, it has been extended to cover high-speed flows with high accuracy $[18,19]$. The discretization of all flow parameters in space was achieved using second-order upwinding. Turbulence throughout the domain was considered using a time or Reynolds-averaged approach due to the quasi-steady nature of the full-flowing nozzle. Air behaving in accordance with the ideal gas law was used as the working fluid, and the inlet stagnation temperature was maintained at $450 \mathrm{~K}$, consistent with the conditions recorded in [15]. Viscosity was modeled using a three-coefficient Sutherland approximation due to the low stagnation enthalpy [20].

To satisfy solution convergence, mass flow rate monitors were set on the nozzle inlet, the exit plane, and the domain outlet boundaries. Solution convergence was accepted when all surface monitor values remained consistent over 500 iterations and continuity was satisfied from the recorded mass flux between the inlet and outlet boundaries. Verification of the numerical model was determined by establishing

Table 3 Geometric parameters in the S1 and S3 nozzle

\begin{tabular}{lcccccccc}
\hline \hline Model & $\overline{r_{t 1}}$ & $\overline{y_{2}}$ & $\theta_{1}$ & $\theta_{2}$ & $\overline{x_{2}}$ & $\overline{r_{m n}}$ & $\overline{x_{n}}$ & $\Delta L, \%$ \\
\hline S1 & 0.500 & 4.472 & 35.03 & 4.000 & 10.44 & 18.93 & 9.660 & -7.370 \\
S3 & 3.000 & 4.271 & 27.00 & 0.000 & 15.75 & 27.01 & 13.62 & -13.49 \\
\hline \hline
\end{tabular}

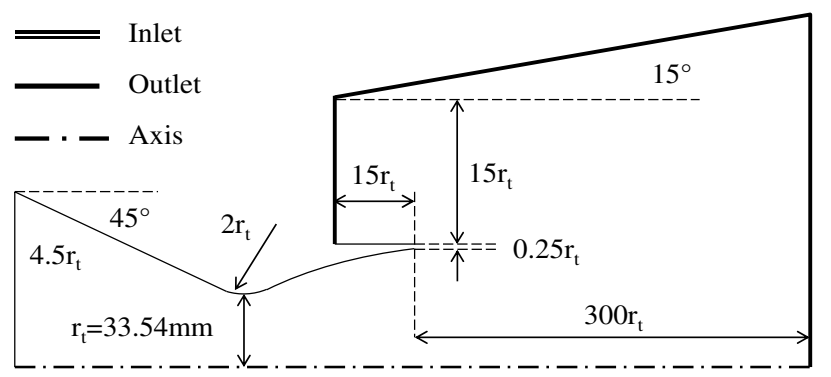

Fig. 5 Numerical domain including inlet and downstream exhaust dimensions (not to scale).

grid independence and ensuring that the selected turbulence model was capable of describing relevant flow effects. The existing [15] S3 nozzle was selected for the verification process because of a higher correlation with the Vulcain nozzle than the S1 design. The verification process was focused on minimizing uncertainty of the calculated thrust at full-flowing conditions and the wall pressure distribution under separated flow conditions. Full-flowing conditions were replicated using a inlet-to-exit pressure ratio (PR) of 50, consistent with existing data [15]. Comparatively, separated flow conditions were generated using a PR of 20 , where a "restricted" shock separation [21] would be expected to occur [15].

\section{A. Geometric Domain}

The computational domain consisted of the convergent-divergent nozzle and a downstream exhaust region. The downstream region was sized to ensure the effect of the domain boundaries on the flowfield was negligible. A throat radius of $33.54 \mathrm{~mm}$ was selected in both the S1 and S3 nozzles, consistent with the existing design [15]. As no information was provided about the convergent section of the nozzle, a constant angle contour at $45 \mathrm{deg}$ was used from the curved throat region. The inlet-to-throat area ratio was sized to ensure the inlet pressure could be assumed to be equal to the total or stagnation pressure with less than $0.5 \%$ error, and flow through the domain was controlled using a pressure inlet and pressure outlet boundary. Figure 5 outlines the dimensions of the model and structure of the numerical domain.

\section{B. Grid}

The computational domain was discretized using a fully structured scheme comprising quadrilateral cells in all models. To determine the effect of the downstream exhaust region on the solution and if computational time could be further reduced, a "short" grid at each density level was used that consisted of the nozzle geometry only. A nondimensional wall distance $y^{+} \overleftarrow{\text { of }}$ one was maintained to ensure the presence of elements within the viscous region of the boundary layer. All calculations were completed using the one-equation SpalartAllmaras (SA) [22] turbulence model. This model was selected due to its proven capability of describing flowfields with shocks due to flow separation [23]. Grid independence was determined from the thrust coefficient at full-flowing conditions and the static wall pressure at separated flow conditions, as shown in Table 4 and Fig. 6 , respectively.

The grid-based numerical uncertainty of the calculated thrust at full-flowing conditions was quantified using Roache's grid convergence index [24] with an applied safety factor of three, and it was found to be less than $1 \%$ in all cases. The influence of a downstream

Table 4 Numerical uncertainty of thrust coefficient at full-flowing conditions (PR = 50)

\begin{tabular}{lrcccc}
\hline \hline Grid & Elements & $C_{F}$ & $E_{C S}, \%$ & $E_{C F}, \%$ & $E_{S F}, \%$ \\
\hline Coarse (C) & $460 \times 135$ & 1.319 & 0.345 & 0.220 & - \\
Standard (S) & $600 \times 180$ & 1.320 & 0.084 & - & 0.675 \\
Fine (F) & $860 \times 220$ & 1.318 & - & 0.027 & 0.112 \\
C short & $160 \times 70$ & 1.319 & 0.179 & 0.000 & - \\
S short & $250 \times 95$ & 1.320 & 0.065 & - & 0.212 \\
F short & $410 \times 120$ & 1.319 & - & 0.000 & 0.069 \\
\hline \hline
\end{tabular}




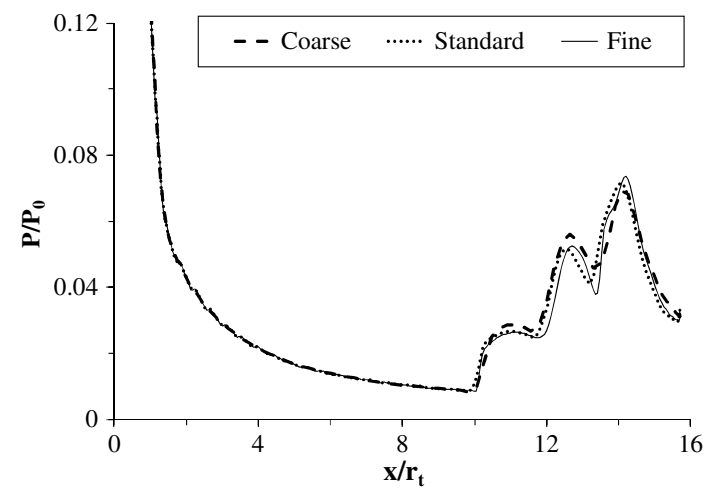

Fig. 6 Effect of grid resolution on static wall pressure $(P R=20)$.

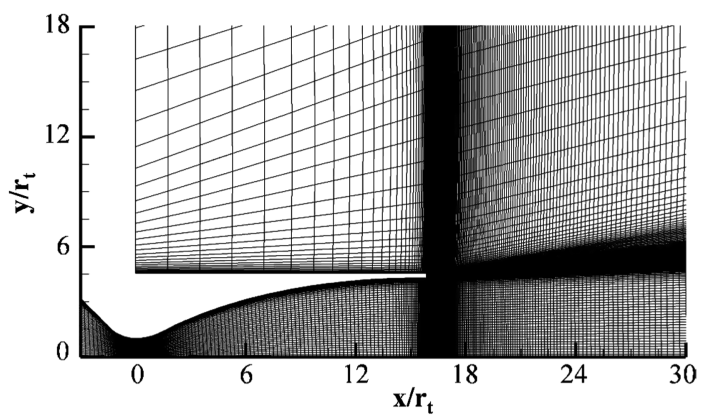

Fig. 7 S3 nozzle coarse grid.

exhaust region was negligible in all cases, and the short coarse grid was therefore selected to calculate the thrust coefficient at all fullflowing conditions ( $\mathrm{PR} \geq 50$ ).

The difference in the location of the separation point was within $1 \%$ between grids, where the characteristics of a restricted shock flowfield was observed in all cases [21]. Evidence of compression waves forming within the nozzle was observed in the static pressure distribution in all grids at $x / r_{t}<4$. This was expected due to the nonideal turning of the flow in a TOP contour. Although the magnitude of the pressure peaks was damped in the coarse and standard grids, the preliminary and comparative nature of the analysis placed a focus on the separation point and general flow characteristics. As the convergence requirements prohibited the use of a short grid under separated flow conditions, the coarse grid was selected for use in the separated flow condition and is shown in Fig. 7 for the S3 nozzle.

\section{Turbulence Modeling}

The selection of an appropriate turbulence closure model was verified by again observing variation in static wall pressure under separated flow conditions and calculation of the thrust coefficient in the full-flowing nozzle. Here, the one-equation SA model, twoequation $k$-omega shear stress transport ( $k-\omega \mathrm{SST})$ [25], and twoequation $k$-epsilon realizable ( $k$ - $\varepsilon$ realizable) [26] models were compared to an inviscid solution. Table $\underline{5}$ and Fig. $\underline{8}$ and outline the difference between calculated thrust and wall pressure, respectively.

The difference between calculated thrust in each of the viscous solutions was negligible, where a $0.5 \%$ increase in thrust coefficient was observed in the inviscid solution. Comparatively, the choice of turbulence model had a significant effect on the wall pressure distribution. The distribution produced using all models was

Table 5 Effect of turbulence model on thrust coefficient $(\mathbf{P R}=\mathbf{5 0})$

\begin{tabular}{lcc}
\hline \hline PR & $C_{F}$ & $\Delta C_{F}, \%$ \\
\hline Inviscid & 1.327 & - \\
SA & 1.319 & 0.607 \\
$k-\varepsilon$ realizable & 1.319 & 0.607 \\
$k-\omega$ SST & 1.319 & 0.607 \\
\hline \hline
\end{tabular}

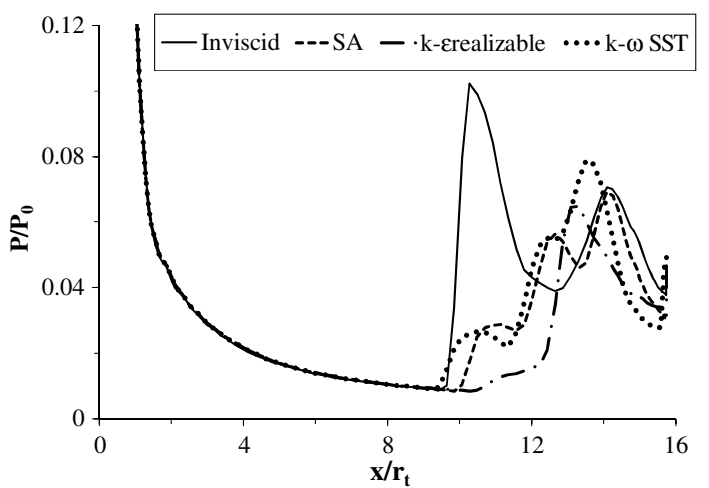

Fig. 8 Effect of turbulence model on wall pressure distribution $(\mathrm{PR}=20)$.

indicative of the restricted shock separation (RSS) flow regime [21]. The pressure distribution indicated that a single separation "ring" was formed in the $k-\varepsilon$ realizable solution compared to two in all other solutions. This coupled with the unconventional distribution immediately downstream of the separation point led the $k-\varepsilon$ realizable model to be discarded as a viable candidate. The RSS phenomenon involves a shallow separated region downstream of the initial separation shock and upstream of where the reflected shock hits the nozzle wall [21]. The pressure in this region is known to be between the critical pressure for separation and the local ambient pressure [21]. This was not adhered to in the inviscid solution; thus, it was discarded as well. The result suggested that both the SA and $k-\omega$ SST turbulence models were capable of describing flow behavior within the nozzle at separated conditions. The SA model was consequently selected to generate all results due to a faster convergence rate compared to the $k-\omega$ SST model.

\section{Results}

Flow parameters for both nozzle configurations were evaluated to ensure equivalent performance, irrespective of the method of contour generation. Numerical results were compared to known values [15] to ensure validation of the numerical model and a further analysis was conducted at a number of pressure ratio conditions to determine the nozzle performance across the entire range of operating regimes expected in a core stage engine.

\section{A. Validation}

To validate the numerical model, nozzle contours generated using the Rao-based TOP method (hereafter referred to as Rao) and the arc method (hereafter referred to as arc) were compared with those designed and validated by Volvo Aero (hereafter referred to as Volvo) as an approximation to the Vulcain nozzle, where the static pressure distribution and equivalent Mach number were used to confirm this approximation [15]. The primary aim of the TOP-based (Rao) nozzle was to ensure the numerical model was capable of adequately predicting flow effects, as behavior would theoretically be identical to the Volvo nozzles. The arc-based contour would give an indication of how flow behavior varied between nozzles designed using the conventional and arc methods. A PR of 50 and inlet stagnation temperature of $450 \mathrm{~K}$ were used for all simulations, consistent with that used in [15]. Figures 9 and 10 outline the static pressure distribution and equivalent Mach number in the S1 and S3 nozzles, respectively.

There was no discernible difference between the Volvo and Rao contours in either the $\mathrm{S} 1$ or $\mathrm{S} 3$ nozzles, confirming that the numerical model was capable of describing flow behavior within the nozzle. The reduced contour length was clearly evident in the arc contour in both the S1 and S3 nozzles. A decrease in the static pressure in the arc nozzle was complimented by an increased Mach number throughout an $x / r_{t}$ of approximately four to eight. This behavior was caused by a lower initial rate of wall turning angle, and therefore continued expansion in the arc-based design. Comparatively, the increased rate 


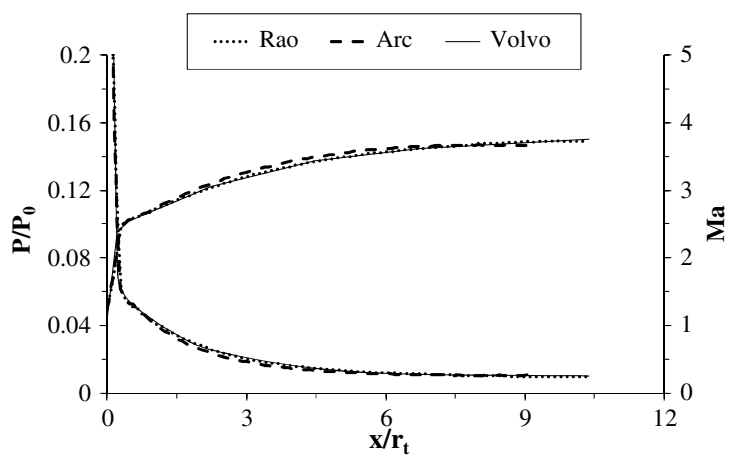

Fig. 9 Comparison with existing data for the S1 nozzle (PR $=50)$.

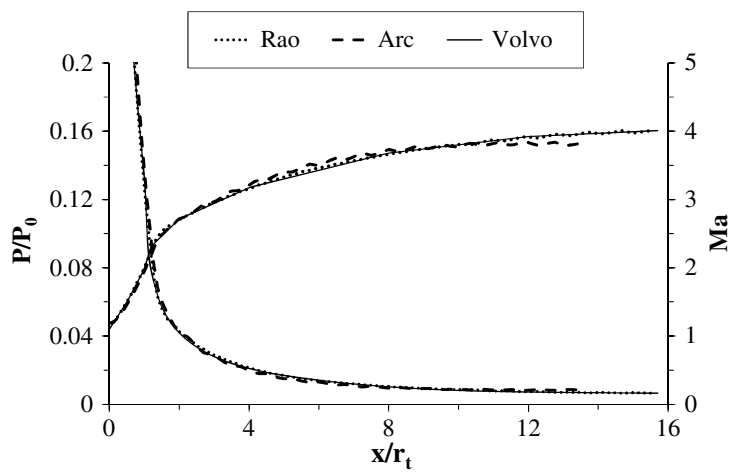

Fig. 10 Comparison with existing data for the S3 nozzle $(P R=50)$.

of flow turning toward the latter half of the arc nozzle was evident from the static pressure and corresponding decrease in Mach number. The greater exit pressure observed in the arc contour may reduce the PR required for full-flowing conditions, and consequently reduce the flow time spent in the transient startup and shutdown phases.

\section{B. Analysis}

The evaluation of both nozzle types required the observation of flow parameters across a number of operating conditions. To achieve this, the receiver pressure was varied to produce a number of pressure ratios that would simulate nozzle behavior across the range of flow conditions expected in a core stage nozzle. It is important to recall that any adverse effects observed in the arc-based design would nullify the geometric advantage previously demonstrated. Differences between the Rao and arc nozzles were compared using static pressure distribution and contours of Mach number at separated conditions and are shown in Figs. 11-14. Comparison at full-flowing conditions was made using the thrust coefficient in the Rao-based nozzle and weighted thrust coefficient in the arc-based nozzle, as shown in Tables $\underline{6}$ and $\underline{7}$.

The onset of flow separation in the Rao nozzle was downstream of the arc contour in both configurations under separated flow conditions. However, when the relative length between the Rao and arc

Table 6 Variation in thrust coefficient in the S1 nozzle

\begin{tabular}{lccc}
\hline \hline $\mathrm{PR}$ & $C_{F}$ Rao & $C_{F, w}$ arc & $\Delta C_{F}, \%$ \\
\hline 50 & 1.314 & 1.319 & 0.343 \\
350 & 1.619 & 1.626 & 0.440 \\
1000 & 1.653 & 1.660 & 0.428 \\
\hline \hline
\end{tabular}

Table 7 Variation in thrust coefficient in the $S 3$ nozzle

\begin{tabular}{lccc}
\hline \hline $\mathrm{PR}$ & $C_{F}$ Rao & $C_{F, w}$ arc & $\Delta C_{F}, \%$ \\
\hline 50 & 1.319 & 1.343 & 1.781 \\
350 & 1.625 & 1.652 & 1.666 \\
1000 & 1.662 & 1.681 & 1.478 \\
\hline \hline
\end{tabular}

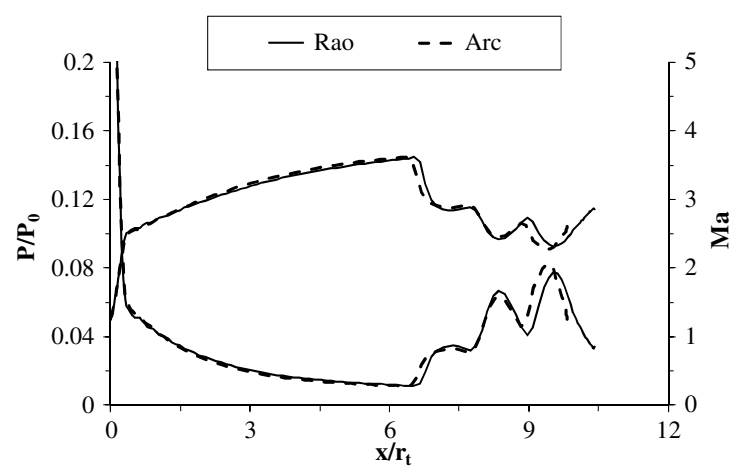

Fig. 11 Pressure and Mach number distributions in the S1 nozzle $(\mathbf{P R}=20)$.

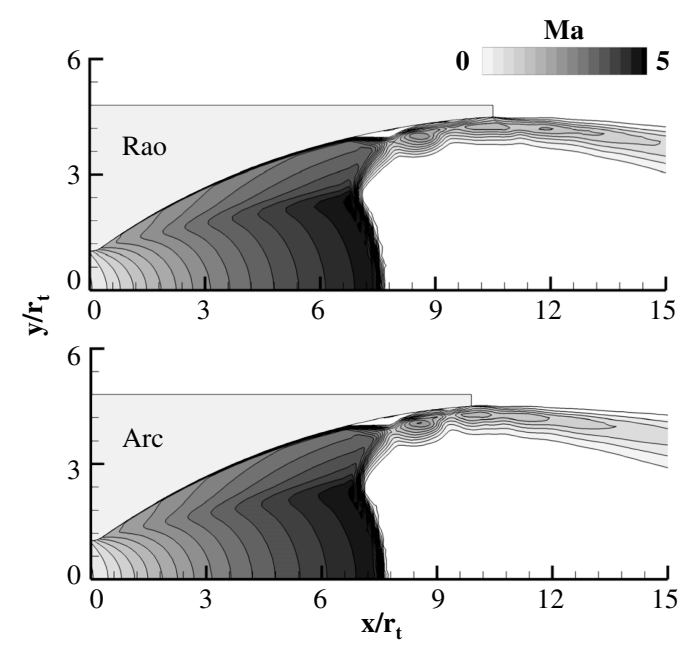

Fig. 12 Contour plot of Mach number in the S1 nozzle $(P R=20)$.

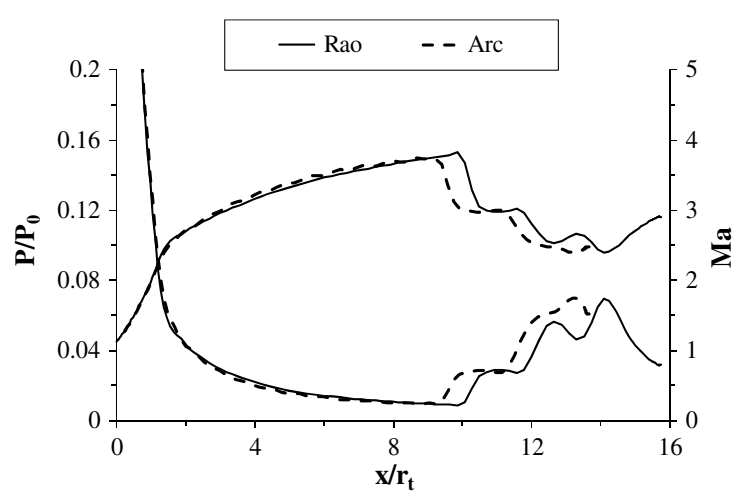

Fig. 13 Pressure and Mach number distributions in the S3 nozzle $(\mathbf{P R}=20)$.

contours is considered, the distance between the separation point and the nozzle exit was reduced in the arc-based design in both configurations. Observation of the magnitude of this offset suggested that it was approximately one-third of the magnitude of the total length reduction in each case, which would imply a reduction in side loading in the arc-based nozzle under separated flow conditions [15]. This benefit would be slightly decreased due to a small increase in the pressure peak observed in the arc-based design. However, the influence of this is difficult to quantify and unlikely to completely offset the lower moment arm evident in the arc-based design.

The main discrepancy in flow structure observed in the attached region between the Rao and arc contours was in the shape of the Mach contours itself. In both cases, the lower initial rate of turning in the arc-based design reduced the severity of the compression wave generated at the inflection between the expansion and turning curves. 


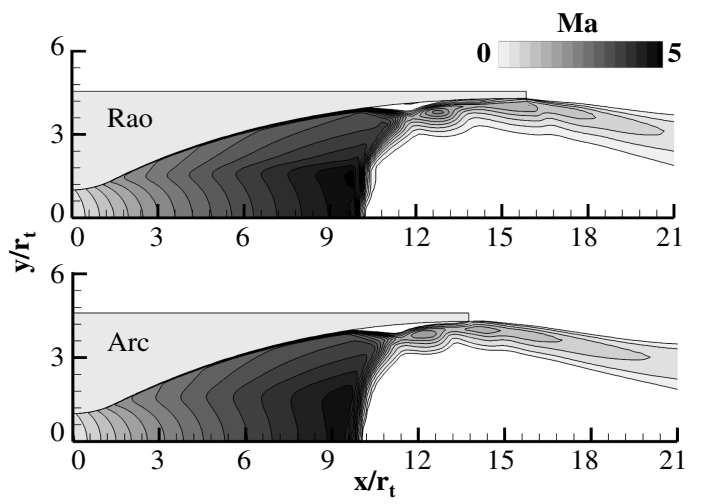

Fig. 14 Contour plot of Mach number in the S3 nozzle (PR = 20).

This effect was evident in both configurations, albeit far more pronounced in the S3 nozzle. The general structure of the separated region was consistent between nozzle designs and configurations. However, the flow structure appeared to be axially compressed in both arc-based nozzles. In both cases, this compression was not equal to the difference in nozzle length and was in agreement with the calculated flow parameters.

The thrust coefficient was calculated at full-flowing overexpanded $(\mathrm{PR}=\$ 50)$, ideal $(\mathrm{PR}=\$ 350)$, and underexpanded $(\mathrm{PR}=\$ 400)$ flow regimes to represent the entire range of operating conditions expected to occur in a core stage nozzle. The thrust-to-weight scaling factor introduced in Eq. (12) was applied to the arc-based design in each case to ensure an accurate comparison between design methods. The calculated thrust coefficient in the arc-based design was greater than the equivalent Rao in all cases, where the magnitude of increase was over $1 \%$ greater in the $\mathrm{S} 3$ configuration. However, the similarity of the S1 nozzle geometric parameters with existing nozzle designs suggests an expected increase in thrust coefficient as a result of the arc-based design on the order of $0.4 \%$.

\section{Transition}

To confirm that a reduction of nozzle length without adverse separation effects was possible, an evaluation of the transition phases in the S1 nozzle was conducted. This configuration was selected due to the availability of experimental transition pressure information in [15] coupled with the slight increase in pressure peaks observed in the separated flow condition in the arc-based design. Nozzle behavior was evaluated at PRs of 14 and 16 to observe flow conditions at the threshold between "free" and restricted shock separation during the transient startup condition [21]. Figures 15-18 outline the static wall pressure distributions and contours of Mach number in the Rao and arc nozzles at each PR.

The correct prediction of a free or restricted separation flow regime and a static pressure distribution that was consistent with the experimental data was observed in the Rao nozzle, providing additional validation of the numerical model for use under separated flow conditions. At a PR of 14, the difference between the numerical and

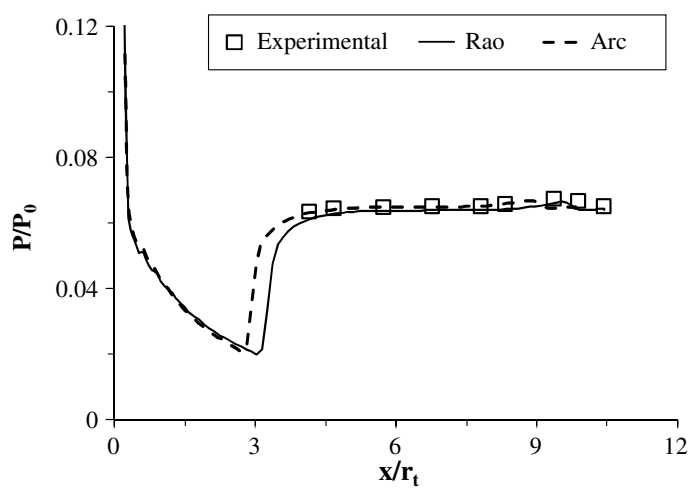

Fig. 15 Wall pressure distribution in the $S 1$ nozzle $(P R=14)$.

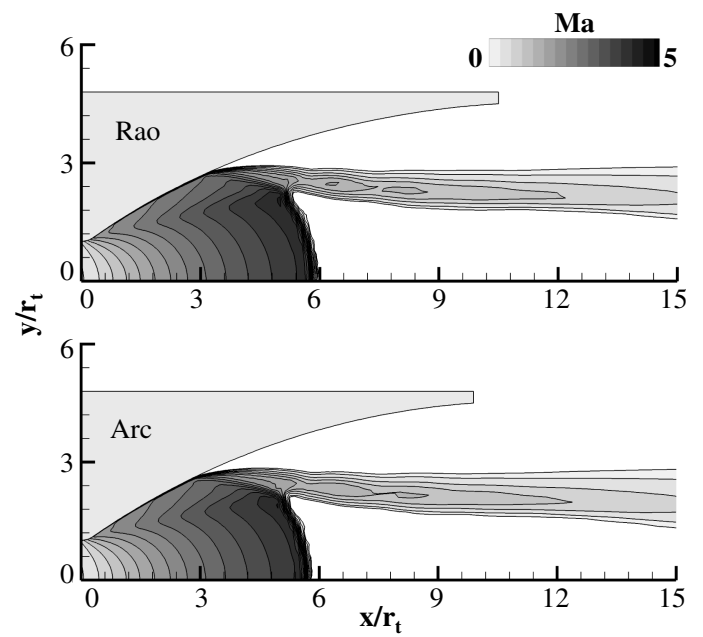

Fig. 16 Contour plot of Mach number in the $S 1$ nozzle $(P R=14)$.

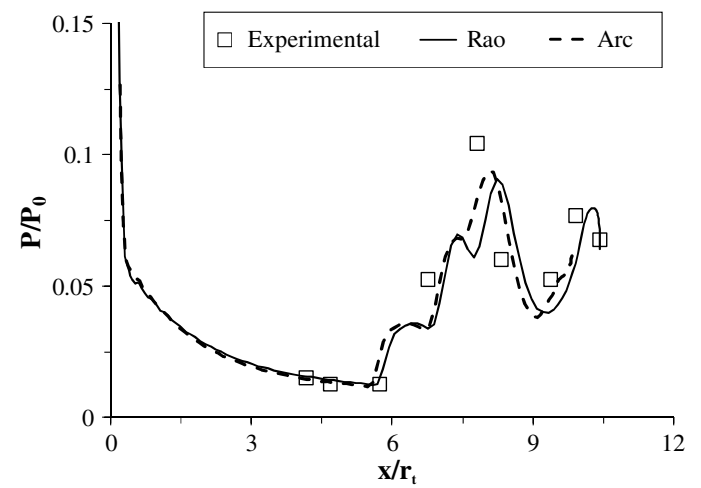

Fig. 17 Wall pressure distribution in the $S 1$ nozzle $(P R=16)$.

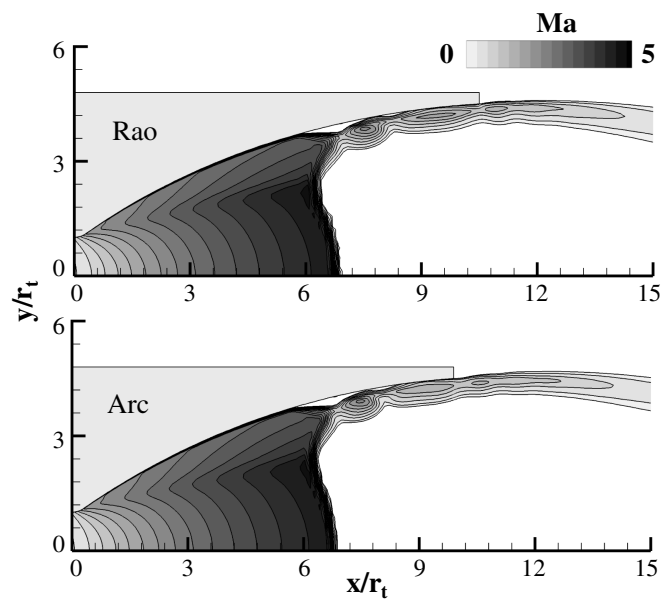

Fig. 18 Contour plot of Mach number in the $S 1$ nozzle $(P R=16)$.

experimental static pressure readings was less than $4 \%$. The main discrepancy between the Rao and arc pressure distributions was a predicted separation point location approximately 5\% upstream in the arc-based contour. An earlier point of separation was expected in the arc nozzle due to the higher initial rate of expansion shown to occur. However, an overall decrease in the relative separation length remained evident in the arc-based contour.

As expected, the restricted shock separation flow phenomenon was predicted in both the Rao and arc nozzles at a PR of 16. The separation location was predicted within $2 \%$ in both nozzle types, where the main discrepancy of the pressure distribution was a lower pressure peak evident in the numerical solution. This result can largely be attributed to the use of a time-averaged solution to a highly 
transient flow phenomenon [21]. Similar to the separated results at a PR of 20, a slight increase in pressure peak was observed in the arc nozzle. However, in all cases, the difference in separation point location and the increase in static wall pressure was minimal compared to the reduction in nozzle length, suggesting that a reduction in side loads may also be possible through use of the arcbased design method.

\section{Conclusions}

Two arc-based and equivalent conventional nozzle contours have been evaluated numerically to compare the flow characteristics and predicted thrust in a high-area-ratio nozzle contour. A comparison of the arc-based contours to existing operational nozzles indicated that an average reduction in length of $7.5 \%$ can be expected when the arcbased method is applied to a core stage nozzle design. The calculated thrust coefficient in the arc-based design was $0.3-1.8 \%$ greater than the equivalent conventional nozzle across the range of full-flowing conditions tested. Additionally, the flow parameters and transition behavior under separated flow conditions suggested equivalent side loading and separated flow characteristics can be expected in the arcbased design. The result warrants further exploration of the arc-based method for the design of a high-area-ratio nozzle.

\section{References}

[1] Malina, F. J., "Characteristics of the Rocket Motor Based on the Theory of Perfect Gases," Journal of the Franklin Institute, Vol. 230, No. 4, 1940, pp. 433-454.

doi:10.1016/S0016-0032(40)91348-5

[2] Shapiro, A. H., "Nozzles for Supersonic Flow Without Shock Fronts," Journal of Applied Mechanics, Vol. 11, No. 2, 1944, pp. 93-100.

[3] Ahlberg, J. H., Hamilton, S., Migdal, D., and Nilson, E. N., "Truncated Perfect Nozzles in an Optimum Nozzle Design," ARS Journal, Vol. 31, No. 5, 1961, pp. 614-620. doi: $10.2514 / 8.5577$

[4] Hoffman, J. D., "Design of Compressed Truncated Perfect Nozzles," Journal of Propulsion and Power, Vol. 3, No. 2, 1987, pp. 150-156. doi: $10.2514 / 3.22967$

[5] Guderley, G., and Hantsch, E., "Beste Formen fur Aschsensymmetrische Uberschallschubdusen," Zeitschrift fur Flugwissenschaften, Vol. 3, No. 9, 1955, pp. 305-313.

[6] Rao, G. V. R., "Exhaust Nozzle Contour for Optimum Thrust," Journal of Jet Propulsion, Vol. 28, No. 6, 1958, pp. 377-382. doi: $10.2514 / 8.7324$

[7] Rao, G. V. R., "Approximation of Optimum Thrust Nozzle Contour," ARS Journal, Vol. 30, No. 6, 1960, p. 561.

[8] Allman, J. G., and Hoffman, J. D., "Design of Maximum Thrust Nozzle Contour by Direct Optimization Methods," AIAA Journal, Vol. 19, No. 6, 1981, pp. 750-751. doi:10.2514/3.50999

[9] Chen, J. S., and Freeman, J. A., "Thrust Chamber Performance Using Navier-Stokes Solution," NASA TR-D951729, 1984.

[10] Sutton, G. P., and Biblarz, O., Rocket Propulsion Elements, 7th ed., Wiley, New York, 2001, pp. 75-81, Chaps. 2, 3 .
[11] Sutton, G. P., History of Liquid Propellant Rocket Engines, AIAA, Reston, VA, 2006, pp. 88-92, Chap. 4.

[12] Rao, G. V. R., "Recent Developments in Rocket Nozzle Configurations," ARS Journal, Vol. 31, No. 11, 1961, pp. 1488-1494. doi: $10.2514 / 8.5837$

[13] Davis, D. K., "Investigation of Optimization Techniques for Solid Rocket Motor Nozzle Contour," 18th Joint Propulsion Conference, AIAA Paper 1982-1188, 1982.

[14] Vuillermoz, P., Weiland, C., Hagemann, G., Aupoix, B., Grosdemange, H., and Bigert, M., "Nozzle Design and Optimisation," Progress in Astronautics and Aeronautics: Liquid Rocket Thrust Chambers, AIAA, Reston, VA, 2004, pp. 469-492, Chap. 13.

[15] Östlund, J., "Flow Processes in Rocket Engine Nozzles with Focus on Flow Separation and Side-Loads," Ph.D. Dissertation, Royal Inst. of Technology, Stockholm, 2002.

[16] Jewett, R. P., and Halchak, J. A., "The Use of Alloy 718 in the Space Shuttle Main Engine," Superalloys 718, 625 and Various Derivatives, Minerals, Metal, and Material Soc., Warrendale, PA, 1991, pp. 749-760.

[17] Kirner, E., Thelemann, D., and Wolf, D., "Development Status of the Vulcain Thrust Chamber," Acta Astronautica, Vol. 29, No. 4, 1993 , pp. 271-282. doi:10.1016/0094-5765(93)90140-R

[18] Kurbatskii, K. A., and Montanari, F., "Application of Pressure-Based Coupled Solver to the Problem of Hypersonic Missiles with Aerospikes," 45th Aerospace Sciences Meeting, AIAA Paper 2007$0462,2007$.

[19] Koutsavdis, E. K., and Stuckert, G., "A Numerical Investigation of the Flow Characteristics of Plug Nozzles Using Fluent," 40th Aerospace Sciences Meeting, AIAA Paper 2002-0511, 2002.

[20] Sutherland, W., "The Viscosity of Gases and Molecular Force," Philosophical Magazine, Vol. 36, No. 223, 1893, pp. 507-531. doi:10.1080/14786449308620508

[21] Frey, M., and Hagemann, G., "Restricted Shock Separation in Rocket Nozzles," Journal of Propulsion and Power, Vol. 16, No. 3, 2000, pp. $478-484$. doi: $10.2514 / 2.5593$

[22] Spalart, P., and Allmaras, S., "A One-Equation Turbulence Model for Aerodynamic Flows," La Recherche Aerospatiale, Vol. 1, No. 5, 1992, pp. 5-21.

[23] Stark, R., and Hagemann, G., "Current Status of Numerical Flow Prediction for Separated Nozzle Flows," 2nd European Conference for Aerospace Sciences, von Karman Inst., EUCASS, Brussels, 2007, Paper 362.

[24] Roache, P. J., "Quantification of Uncertainty in Computational Fluid Dynamics," Annual Review of Fluid Mechanics, Vol. 29, Jan. 1997, pp. $123-160$. doi:10.1146/annurev.fluid.29.1.123

[25] Menter, F. R., "Two Equation Eddy-Viscosity Turbulence Models for Engineering Applications," AIAA Journal, Vol. 32, No. 8, 1994, pp. $1598-1605$. doi:10.2514/3.12149

[26] Shih, T. H., Liou, W. W., Shabbir, A., and Zhu, J., "A New $k-\epsilon$ EddyViscosity Model for High Reynolds Number Turbulent Flows: Model Development and Validation," Computers Fluids, Vol. 24, No. 3, 1995, pp. 227-238.

K. Frendi

Associate Editor 